\title{
Increased portal vein diameter is predictive of portal vein thrombosis development in patients with liver cirrhosis
}

\author{
Gang Dong ${ }^{1}$, Xiao-Quan Huang ${ }^{1}$, Yu-Li Zhu ${ }^{2}$, Hong Ding ${ }^{2}$, Feng Li ${ }^{1}$, Shi-Yao Chen ${ }^{1,3}$ \\ ${ }^{1}$ Department of Gastroenterology and Hepatology, Zhongshan Hospital, Fudan University, Shanghai, China; ${ }^{2}$ Department of Ultrasound, \\ Zhongshan Hospital, Fudan University, Shanghai, China; ${ }^{3}$ Endoscopy Center and Endoscopy Research Institute, Zhongshan Hospital, Fudan \\ University, Shanghai, China \\ Contributions: (I) Conception and design: SY Chen, G Dong, XQ Huang; (II) Administrative support: SY Chen, F Li; (III) Provision of study \\ materials or patients: G Dong, XQ Huang; (IV) Collection and assembly of data: YL Zhu, H Ding; (V) Data analysis and interpretation: G Dong, \\ XQ Huang; (VI) Manuscript writing: All authors; (VII) Final approval of manuscript: All authors. \\ Correspondence to: Shi-Yao Chen. Department of Gastroenterology and Hepatology, Zhongshan Hospital, Fudan University, Shanghai 200032, China. \\ Email: Chen.shiyao@zs-hospital.sh.cn.
}

\begin{abstract}
Background: Cirrhotic patients with portal vein thrombosis (PVT) may have a high risk of hepatic decompensation and increased mortality. This study aimed to investigate if increased portal vein diameter is associated with PVT development.

Methods: A total of 174 cirrhotic patients were enrolled between February 1 and August 31, 2017. All participants were divided into PVT (n=62) and non-PVT (n=112) groups based on the thrombus that was detected by ultrasonography and confirmed by computed tomography angiography (CTA).

Results: The study participants, aged $54.7 \pm 10.5$ years (PVT) and $55.8 \pm 11.6$ years (non-PVT), were included in this analysis. The Child-Pugh score of PVT or non-PVT was $6.6 \pm 1.3$ and $5.8 \pm 0.9$, respectively. Hepatitis B virus (HBV) is the primary etiological agent of cirrhosis. Logistic regression, receiver operating characteristic (ROC), and nomograph analysis designated portal diameter as the strongest independent risk factor for predicting PVT development [odds ratio (OR): 3.96, area under the ROC curve (AUC): 0.88; $\mathrm{P}<0.01]$, and the cutoff with predictive value for PVT development was $>12.5 \mathrm{~mm}$. No differences were observed in the overall survival (OS) in cirrhosis with or without PVT or stratifying on portal diameter based on the cutoff value.
\end{abstract}

Conclusions: Increased portal diameter is associated with an increased risk of PVT development. Patients with cirrhosis and increased portal diameter are a high-risk subgroup that may need thromboprophylaxis.

Keywords: Cirrhosis; thrombosis; portal vein diameter

Submitted Jun 24, 2020. Accepted for publication Nov 27, 2020.

doi: $10.21037 /$ atm-20-4912

View this article at: http://dx.doi.org/10.21037/atm-20-4912

\section{Introduction}

Portal vein thrombosis (PVT) is a major complication in patients with liver cirrhosis and $<1 \%$ in the general population. However, $0.6-15.8 \%$ of the cirrhosis patients suffered from PVT, varied prevalence PVT according to different studies (1). Several studies showed that PVT was associated with poor outcomes of cirrhosis patients, such as increasing hepatic decompensation, transplantation mortality, and hepatic artery thrombosis (2-4).
The potential mechanism of the development of PVT in cirrhosis patients is not yet clarified, and the occurrence of PVT is a multifactorial process involving inherited and acquired thrombotic risk factors (5). Cirrhosis and portal hypertension induced hepatic structural derangement, slowed portal vein blood flow, damaged the vessel wall, and induced hypercoagulability, which served as vital roles in PVT development $(6,7)$. As shown in Figure S1, PVT increased the thickness of spleen. 
PVT is primarily detected and diagnosed by imaging examination, especially computed tomography angiography (CTA) (8). However, due to the high cost and damage to the body, CTA is not suitable for high-risk population screening. Thus, ultrasonography is widely used for screening of high-risk populations with PVT with a diagnostic accuracy of $88-98 \%$ (9). This method is economical, practical, portable, and harmless, and some parameters of the portal vein, such as portal vein diameter, blood flow velocity, and filling defect, could be measured. The blood flow velocity in the portal vein has been reported to predict PVT development (7). However, the effect of portal vein diameter on the formation of PVT in cirrhotic patients is largely unknown. Studies have demonstrated that portal vein dilatation tends to be continuous in cirrhosis (10). Then, the dilated portal vein induces endothelial cell damage (11), which is one of the key factors for thrombosis. Nonetheless, only a few reports are available about the association between portal diameter and PVT development in liver cirrhosis. Therefore, the present study aimed to evaluate the effect of portal diameter on PVT development and prognosis in cirrhotic patients.

We present the following article in accordance with the TRIPOD reporting checklist (available at http://dx.doi. org/10.21037/atm-20-4912).

\section{Methods}

This study complied with the guidelines of the Declaration of Helsinki (as was revised in 2013) and was approved by the Medical Ethics Committee of Zhongshan Hospital, Fudan University, China (No. B2015-133R). Because of the retrospective nature of the study, the requirement for informed consent was waived.

\section{Patients}

From February-August in 2017, 174 patients diagnosed with liver cirrhosis, screened by CT or magnetic resonance (MR), and underwent ultrasonography of the portal venous system, were enrolled. Patients with esophagogastric varices detected by CT, MRI or ultrasonography will be further examined by upper endoscopy to evaluate its severity and decide the need for intervention. The exclusion criteria were as follows: (I) malignant tumor; (II) active hemorrhage; (III) acute infections; (IV) receiving antiplatelet or anticoagulant therapy; (V) a history of abdominal surgery including splenectomy. Subsequently, the patients were divided into the PVT and non-PVT groups based on CTA. All participants were followed up until April 2020.

\section{Blood collection and analysis}

Blood samples were withdrawn after $12 \mathrm{~h}$ of overnight fasting. Platelets count, D-dimer, total cholesterol, triglyceride, and LDL levels, and other biochemical parameters, such as hepatic function, were measured.

\section{Ultrasound with hepatic portal doppler}

After fasting for $>8 \mathrm{~h}$, participants lay supine, breathed normally, kept quiet, and underwent color Doppler ultrasonic diagnosis. The angle between the portal vein and ultrasonic beam was $\angle 60^{\circ}$, and all measurements were conducted using a 2.0-5.0 MHZ convex transducer (iU22 Ultrasound System; Philips Healthcare, Reedsville, PA, USA). The diameters and velocities of the portal vein were measured using B-type ultrasonography and Doppler examination, respectively.

\section{Statistical analysis}

Continuous variables are reported as a mean \pm standard deviation, and categorical data are expressed as numbers and proportions. Statistical analysis was performed using Student's unpaired $t$-test or Mann-Whitney test for comparisons of continuous variables between groups and corrected chi-square test or Fisher's exact test was used for categorical data. Logistic regression models were constructed to assess risk factors for the development of PVT. KruskalWallis test was used to identify the correlation between portal diameter and etiology or thrombus range, respectively. The sensitivity and specificity of risk factors in predicting PVT were evaluated by receiver operating characteristic (ROC) curves, and the risk was assessed by a nomograph. The Kaplan-Meier analysis was used to estimate the rate of overall survival (OS) in patients with and without PVT according to stratified portal diameter. $\mathrm{P}<0.05$ was considered statistically significant. All analyses were performed using SPSS 24.0 (SPSS Inc., Chicago, IL, USA).

\section{Results}

\section{Participant characteristics}

The mean age of the study participants was $54.7 \pm 10.5$ years 
Table 1 Baseline characteristics

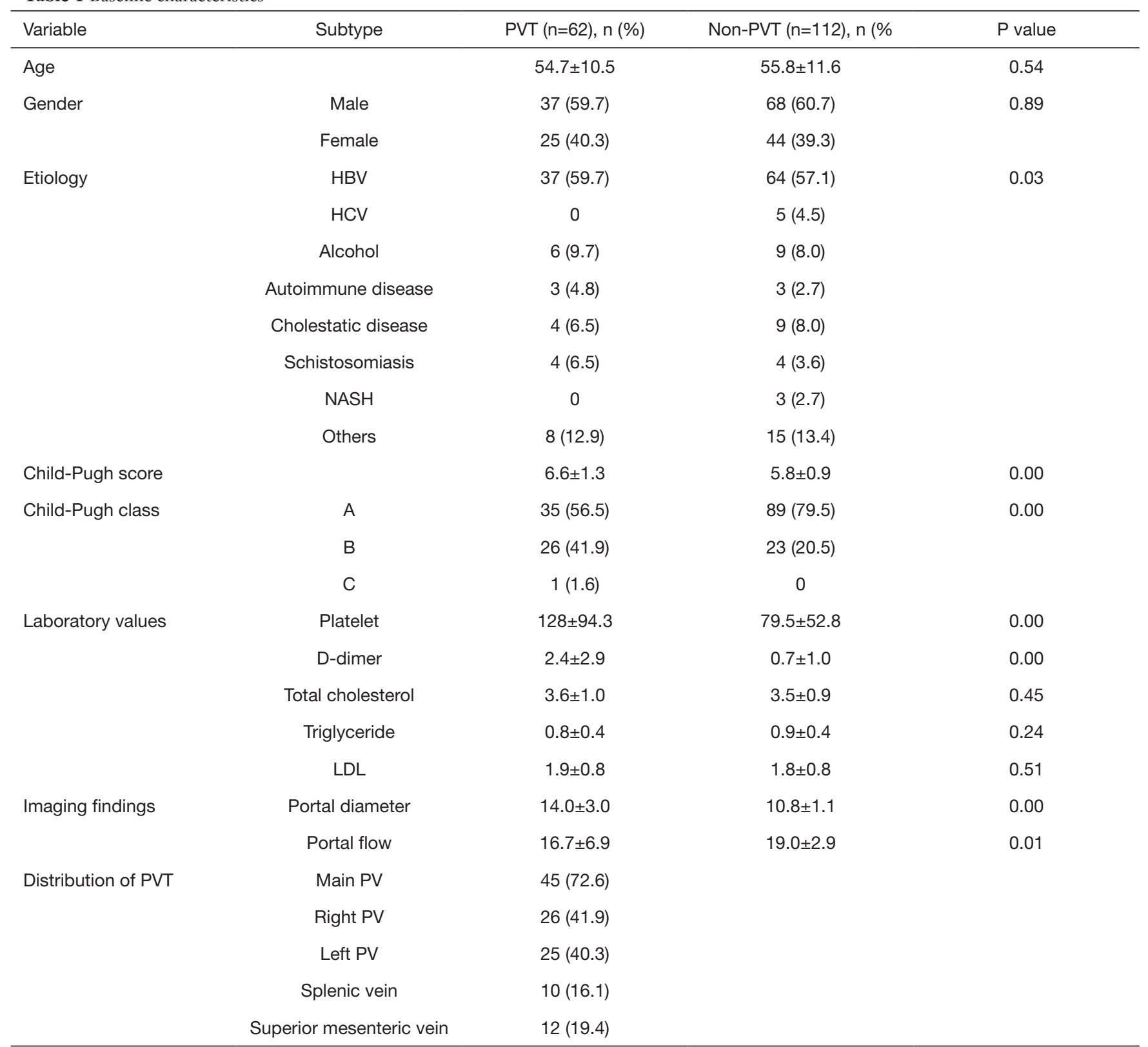

PV, portal vein; PVT, portal vein thrombosis; LDL, low-density lipoprotein.

(PVT) and 55.8 \pm 11.6 years (non-PVT). The cohort consisted of $59.7 \%$ males in PVT and $60.7 \%$ males in nonPVT. Among these patients, hepatitis B virus (HBV) is the major cause of cirrhosis (PVT 59.7\% vs. non-PVT 57.1\%). The Child-Pugh score of PVT and non-PVT was $6.6 \pm 1.3$ and $5.8 \pm 0.9(\mathrm{P}=0.004)$. As shown in Table 1, platelet count and D-dimers examined in the laboratory were $128 \pm 94.3$ (PVT), $79.5 \pm 52.8$ (non-PVT) and 2.4 \pm 2.9 (PVT), $0.7 \pm 1.0$
(non-PVT), respectively. Also, total cholesterol (PVT, 3.6 \pm 1.0 ; non-PVT, $3.5 \pm 0.9$ ), triglyceride (PVT, $0.8 \pm 0.4$; non-PVT, $0.9 \pm 0.4$ ), and LDL levels (PVT, $1.9 \pm 0.8$; nonPVT, $1.8 \pm 0.8)$ were detected. Portal diameter and portal flow detected by ultrasonography were $14.0 \pm 3.0 \mathrm{~mm}$ (PVT), $10.8 \pm 1.1 \mathrm{~mm}$ (non-PVT) and $16.7 \pm 6.9 \mathrm{~cm} / \mathrm{s}(\mathrm{PVT})$, $19.0 \pm 2.9 \mathrm{~cm} / \mathrm{s}$ (non-PVT), respectively, and PVT was distributed in the main portal vein $(72.6 \%)$. 
Table 2 Risk factors in patients with and without portal vein thrombosis

\begin{tabular}{lcccc}
\hline Risk factors & Odds ratio & P value & Std. Err. & 95\% Conf. interval \\
\hline Portal diameter & 3.96 & 0.00 & 1.06 & $2.34-6.70$ \\
Portal flow & 0.85 & 0.01 & 0.05 & $0.76-0.96$ \\
Platelet & 1.02 & 0.00 & 0.00 & $1.01-1.03$ \\
D-dimer & 2.38 & 0.00 & 0.57 & $1.49-3.81$ \\
Child-Pugh score & 1.55 & 0.14 & 0.45 & $0.87-2.75$ \\
\hline
\end{tabular}
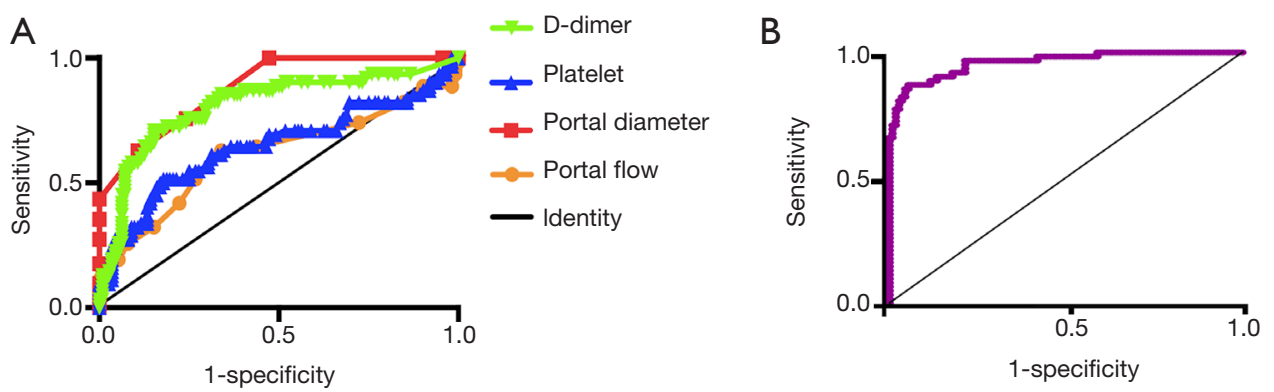

Figure 1 ROC analysis of portal diameter, portal flow, platelet count, D-dimer, and Child-Pugh score (A) or their combination (B) predicting the presence of PVT in cirrhotic patients. Portal diameter was the strongest independent risk factor predicting PVT development. ROC, receiver operating characteristic; PVT, portal vein thrombosis.

Table 3 Performance of risk factors in the diagnosis of PVT

\begin{tabular}{lcccc}
\hline Risk factors & AUC & Sensitivity, \% & Specificity, \% & Cut-off \\
\hline Portal diameter & 0.88 & 63 & 89 & 66 \\
Portal flow & 0.62 & 63 & 82 & 12.50 \\
Platelet & 0.65 & 52 & 85 & 111.50 \\
D-dimer & 0.81 & 71 & 95 & 0.94 \\
Above factors combination & 0.91 & 87 & 0.47 \\
\hline
\end{tabular}

PVT, portal vein thrombosis; AUC, area under curve.

\section{Role of portal diameter on PVT development}

Logistic regression analysis revealed that portal diameter, portal flow, platelet, and D-dimer were identified as independent risk factors for PVT $(\mathrm{P}<0.05)$, and the effect of the Child-Pugh score was rendered insignificant (Table 2). The area under the ROC (AUC) of the portal diameter for PVT development was 0.88 (Figure 1A), which was significant among risk factors, and the sensitivity and specificity for portal diameter in predicting PVT development were $63 \%$ and $89 \%$, respectively (Table 3). On the other hand, the AUC of the combination of the above factors for PVT development was 0.91 (Figure 1B), and the sensitivity and specificity for portal diameter in predicting PVT development were $87 \%$ and $95 \%$, respectively (Table 3), which was consistent with the assessment by nomograph (Figure 2). Cirrhosis could result from a variety of etiologies, which has no influence on the portal diameter and was unaffected by the range of thrombus (Figure 3). Based on the cutoff of portal diameter (Table 3), the PVT participants were divided into two groups: $\leq 12.5$ and $>12.5 \mathrm{~mm}$. No differences were detected in the OS while classifying according to the portal diameter during followup; also, the OS was similar in patients with and without 


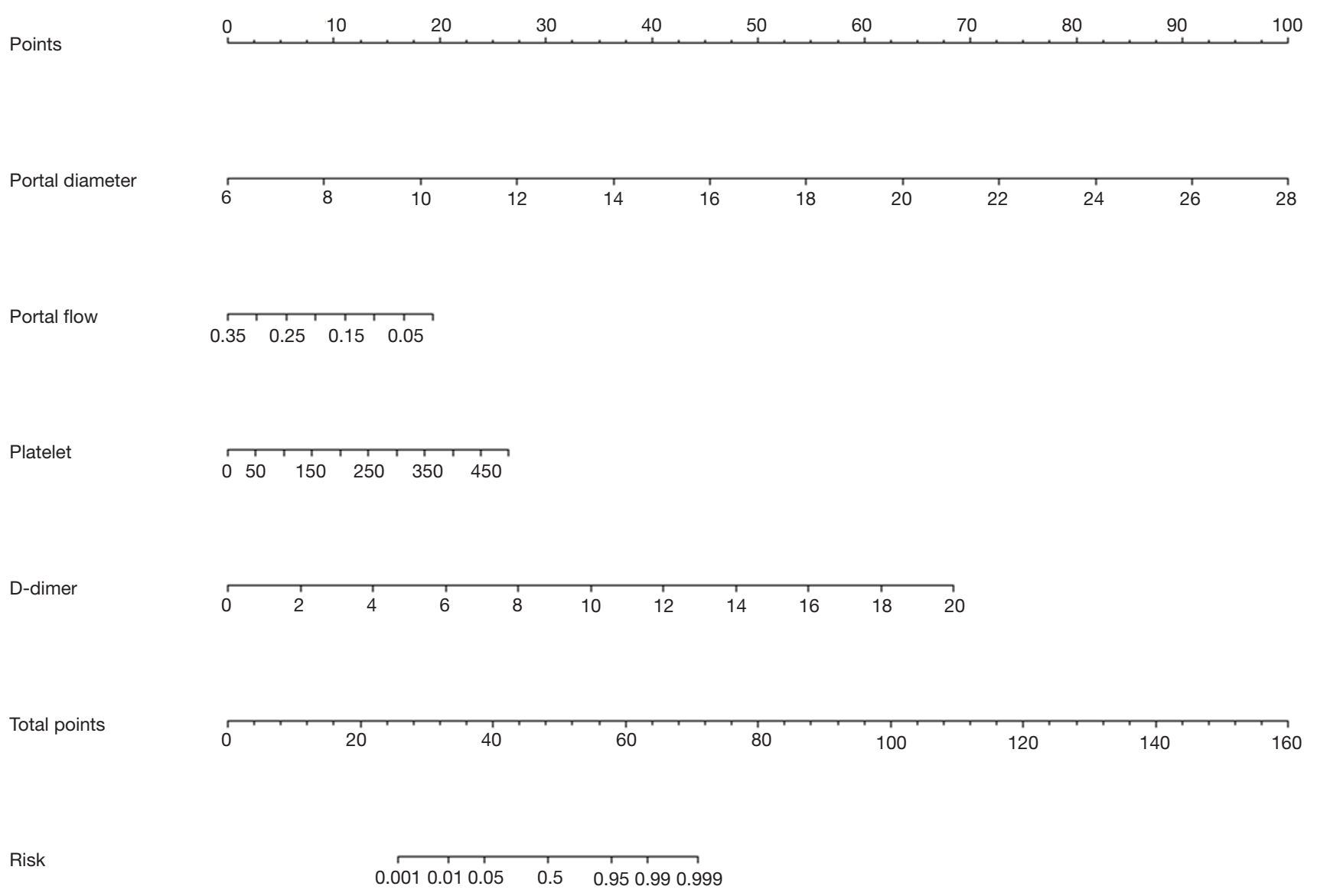

Figure 2 A nomograph for estimating the risk of portal diameter, portal flow, platelet count, and D-dimer in predicting PVT development. Portal diameter was the strongest independent risk factor predicting PVT development. PVT, portal vein thrombosis.
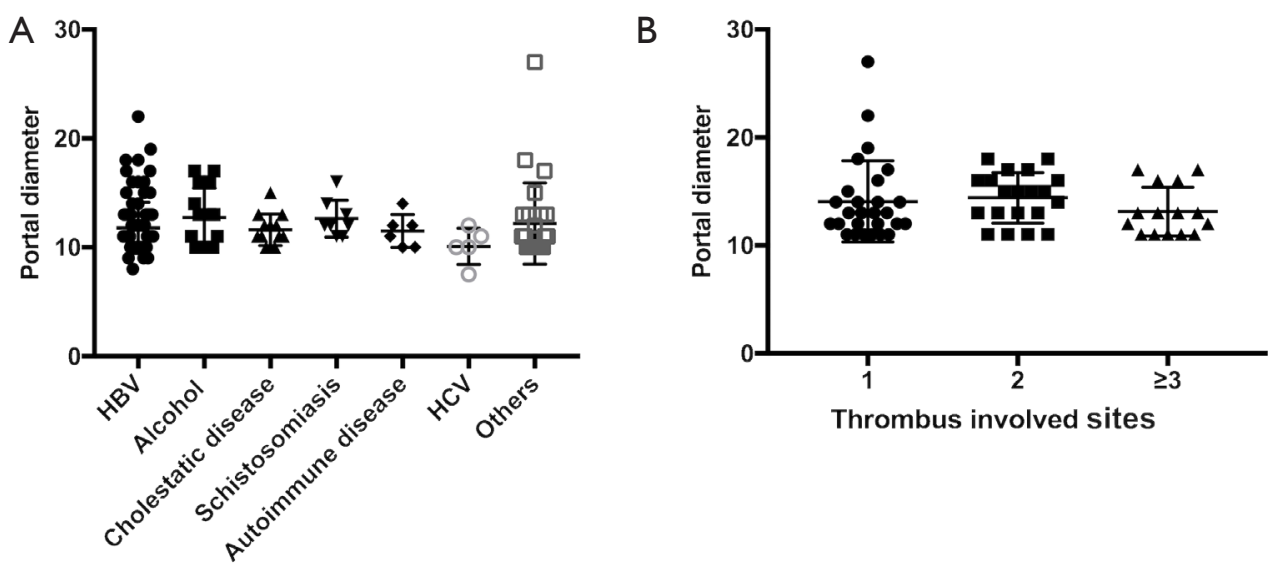

Figure 3 The effect of etiology (A) and the range of thrombus (B) on portal diameter. The range of thrombus included five sites: main portal vein (main PV), left portal vein (left PV), right portal vein (right PV), superior mesenteric vein, and splenic vein. Number of involved sites were divided into three groups: 1 (one site), 2 (two sites), $\geq 3$ (three sites). PV, portal vein. 

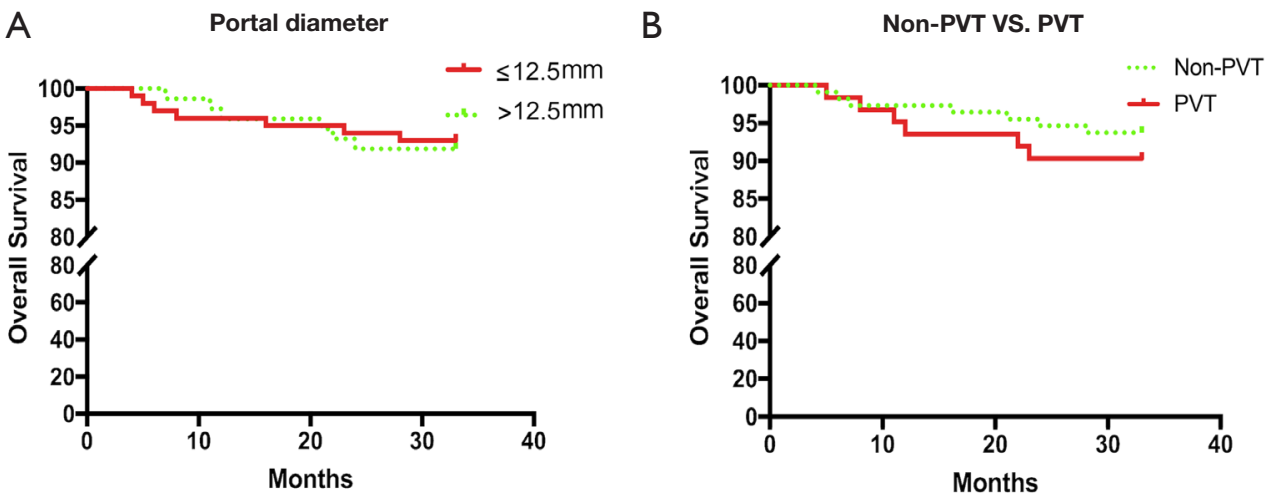

Figure 4 Kaplan-Meier OS curve was analyzed in patients with the stratified portal diameter with and without PVT. (A) No differences were observed in the OS when stratifying by portal diameter. (B) OS was similar in cirrhosis patients with and without PVT. OS, overall survival; PVT, portal vein thrombosis.

PVT (Figure 4).

\section{Discussion}

In the present study, 174 cirrhosis patients with or without PVT were investigated. The results established a positive association between portal diameter and PVT occurrence in cirrhosis patients, and compared it to portal flow, platelet count, and D-dimer; patients with increased portal diameter may be at a high risk to PVT development. This study also confirmed the cutoff of portal diameter as $>12.5 \mathrm{~mm}$, which was sensitive for discerning the cirrhosis patients at risk for PVT development. Based on the cutoff, B-ultrasonography determining the portal diameter should be considered a potential screening tool for identifying patients at the highest risk of thrombosis.

$\mathrm{HBV}$ has been identified as the most frequent cause of cirrhosis and considered as a risk factor for PVT $(12,13)$. In this study, the etiology was HBV in cirrhosis patients with or without PVT. In addition, several other risk factors have been reported to play a role in the development of PVT in cirrhosis (14-16). Since the majority of PVT has no obvious early symptoms, it could be missed diagnosed or be misdiagnosed; subsequently, PVT severely affects the prognosis of cirrhosis patients, including increasing the bleeding risk of varices and liver transplantation mortality (2-4). Therefore, it is crucial to screen out the most valuable risk factors to predict the early development of PVT. Reportedly, the development of PVT in cirrhosis is multifactorial, such as unbalanced coagulation and anti-coagulation activity contributing towards the hypercoagulable state, blood flow stasis from portal hypertension, synergistic interaction of vessel wall, and endothelial injury (6). Elevated blood lipid levels are related to venous thrombosis (17); however, in this study, the correlation between blood lipid levels and PVT was not significant (data not shown), and portal flow, platelet count, Child-Pugh score, D-dimer, and portal diameter were further screened to evaluate their roles in predicting PVT development. As shown in Table 2, an inverse correlation was established between portal flow and PVT occurrence and slow blood flow increased the contact between platelet and vessel wall, which was beneficial to thrombosis, and flow rates $<15 \mathrm{~cm} / \mathrm{s}$ were associated with increased PVT development (7); nevertheless, the cutoff in this study was $18.5 \mathrm{~cm} / \mathrm{s}$. The other risk factor was platelet count, the association between platelet count and PVT was statistically significant, and the cutoff value was 111.5 . Increased platelet indices also contribute to the prethrombotic state in liver cirrhosis and that large platelet may play a specific role in thrombosis (18). Contrary to the previous report (19), we did not find a significant association between child score and PVT development in the present study. The Child-Pugh score is commonly used in clinical practice for quantitative assessment of liver reserve function in patients with liver cirrhosis; the higher the score, the worse the liver reserve function, and the reported incidence of PVT in advanced liver disease increased up to $40 \%$ (20). However, this could be ascribed to the Child-Pugh score A or B in the current study population with adequate liver reserve function; hence, our experimental results were inconsistent with previous studies. D-dimer is a major indicator of 
thrombosis, and detection of D-dimer is primarily used in the prediction and diagnosis of venous thrombosis $(21,22)$. in this study, D-dimer was compared to the portal diameter to predict the PVT development. Cirrhosis has been reported to induce continuous portal vein dilation, which would further damage endothelial cells and then promote the formation of thrombus $(10,11)$. To date, few relevant clinical studies are available about the association between portal diameter and PVT development in cirrhosis. Herein, we found a significant association between portal diameter and PVT, and AUC was higher than other risk factors, screening high-risk patients with PVT by ultrasonography, and intervening early will improve the prognosis of cirrhosis. HBV is one of the risk factors of PVT (6). However, the portal diameter was not related to the etiology, and the range of PVT involvement had little effect on the portal diameter.

A previous study demonstrated that the mortality in PVT patients was increased (23); however, in the current study, no differences were detected in the survival time in cirrhosis patients with or without PVT. This phenomenon could be explained by the fact that the majority of the enrolled patients were Child-Pugh A or B, and the liver function was compensatory. Additionally, endoscopic treatment to prevent rebleeding and other complications, anticoagulant therapy, and antiviral therapy are critical factors that improve the prognosis of PVT patients. Nevertheless, the limitations of the current study include the small sample size, short follow-up period, and data from a single center. These factors might affect the survival time in cirrhosis patients with or without PVT. Thus, a long-term followup of a large sample is essential for an objective evaluation of PVT in the role of the survival of patients with cirrhosis. Moreover, no difference was detected in the survival rates when stratifying portal diameter to those with $\leq 12.5 \mathrm{vs}$. $>12.5 \mathrm{~mm}$ at follow-up.

\section{Conclusions}

In conclusion, the increased portal diameter is associated with significantly increased risk for the development of PVT, and detection of portal diameter $>12.5 \mathrm{~mm}$ is highly sensitive for predicting PVT development. Additional studies are required to confirm the above results, identify additional risk factors in large sample size, and investigate the potential role of thromboprophylaxis for the suppression of PVT development in the high-risk population.

\section{Acknowledgments}

Funding: This study was supported by the fund of clinical research of Zhongshan Hospital, Fudan University (No. 2016ZSLC08) and partly supported by the Innovation Fund of Science and Technology Commission of Shanghai Municipality (No. 19411970200) and Shanghai Sailing Program (No. 19YF1406500).

\section{Footnote}

Reporting Checklist: The authors have completed the TRIPOD reporting checklist. available at: http://dx.doi. org/10.21037/atm-20-4912

Data Sharing Statement: Available at: http://dx.doi. org/10.21037/atm-20-4912

Conflicts of Interest: All authors have completed the ICMJE uniform disclosure form (available at: http://dx.doi. org/10.21037/atm-20-4912). The authors have no conflicts of interest to declare.

Ethical Statement: The authors are accountable for all aspects of the work in ensuring that questions related to the accuracy or integrity of any part of the work are appropriately investigated and resolved. This study complied with the guidelines of the Declaration of Helsinki (as was revised in 2013) and was approved by the Medical Ethics Committee of Zhongshan Hospital, Fudan University (No. B2015-133R). Because of the retrospective nature of the study, the requirement for informed consent was waived.

Open Access Statement: This is an Open Access article distributed in accordance with the Creative Commons Attribution-NonCommercial-NoDerivs 4.0 International License (CC BY-NC-ND 4.0), which permits the noncommercial replication and distribution of the article with the strict proviso that no changes or edits are made and the original work is properly cited (including links to both the formal publication through the relevant DOI and the license). See: https://creativecommons.org/licenses/by-nc-nd/4.0/.

\section{References}

1. Amitrano L, Guardascione MA, Brancaccio V, et al. Risk factors and clinical presentation of portal vein thrombosis 
in patients with liver cirrhosis. J Hepatol 2004;40:736-41.

2. Rodriguez-Castro KI, Porte RJ, Nadal E, et al. Management of nonneoplastic portal vein thrombosis in the setting of liver transplantation: a systematic review. Transplantation 2012;94:1145-53.

3. Stine JG, Shah PM, Cornella SL, et al. Portal vein thrombosis, mortality and hepatic decompensation in patients with cirrhosis: A meta-analysis. World J Hepatol 2015;7:2774-80.

4. Stine JG, Pelletier SJ, Schmitt TM, et al. Pre-transplant portal vein thrombosis is an independent risk factor for graft loss due to hepatic artery thrombosis in liver transplant recipients. HPB (Oxford) 2016;18:279-86.

5. Shetty S, Ghosh K. Thrombophilic dimension of Budd chiari syndrome and portal venous thrombosis--a concise review. Thromb Res 2011;127:505-12.

6. Cagin YF, Bilgic Y, Berber I, et al. The risk factors of portal vein thrombosis in patients with liver cirrhosis. Exp Ther Med 2019;17:3189-94.

7. Stine JG, Wang J, Shah PM, et al. Decreased portal vein velocity is predictive of the development of portal vein thrombosis: A matched case-control study. Liver Int 2018;38:94-101.

8. Qi X, Han G, He C, et al. CT features of non-malignant portal vein thrombosis: a pictorial review. Clin Res Hepatol Gastroenterol 2012;36:561-8.

9. Berzigotti A, Garcia-Criado A, Darnell A, et al. Imaging in clinical decision-making for portal vein thrombosis. Nat Rev Gastroenterol Hepatol 2014;11:308-16.

10. Rector WG, Campra J, Ralls PW, et al. Utility and limitations of splanchnic venous ultrasonography in diagnosis of portal hypertension. J Clin Ultrasound 1986;14:689-96.

11. Dong G, Huang X, Jiang S, et al. Simvastatin Mitigates Apoptosis and Transforming Growth Factor-Beta Upregulation in Stretch-Induced Endothelial Cells. Oxid Med Cell Longev 2019;2019:6026051.

12. van Bommel F, Berg T. Treatment of HBV related

Cite this article as: Dong G, Huang XQ, Zhu YL, Ding H, $\mathrm{Li} \mathrm{F}$, Chen SY. Increased portal vein diameter is predictive of portal vein thrombosis development in patients with liver cirrhosis. Ann Transl Med 2021;9(4):289. doi: 10.21037/atm$20-4912$ cirrhosis. Liver Int 2013;33 Suppl 1:176-81.

13. Lertpipopmetha K, Auewarakul CU. High incidence of hepatitis B infection-associated cirrhosis and hepatocellular carcinoma in the Southeast Asian patients with portal vein thrombosis. BMC Gastroenterol 2011;11:66.

14. Intagliata NM, Caldwell SH, Tripodi A. Diagnosis, Development, and Treatment of Portal Vein Thrombosis in Patients With and Without Cirrhosis. Gastroenterology 2019;156:1582-99.

15. Violi F, Lip GY, Cangemi R. Endotoxemia as a trigger of thrombosis in cirrhosis. Haematologica 2016;101:e162-e163.

16. Kinjo N, Kawanaka H, Akahoshi T, et al. Portal vein thrombosis in liver cirrhosis. World J Hepatol 2014;6:64-71.

17. Doggen CJ, Smith NL, Lemaitre RN, et al. Serum lipid levels and the risk of venous thrombosis. Arterioscler Thromb Vasc Biol 2004;24:1970-5.

18. Girleanu I, Trifan A, Cojocariu C, et al. Platelet indices in patients with de novo portal vein thrombosis and liver cirrhosis. Rev Med Chir Soc Med Nat Iasi 2013;117:641-7.

19. Mikula T, Kozlowska J, Stanczak W, et al. Serum ADAMTS-13 Levels as an Indicator of Portal Vein Thrombosis. Gastroenterol Res Pract 2018;2018:3287491.

20. Villa E, Camma C, Marietta M, et al. Enoxaparin prevents portal vein thrombosis and liver decompensation in patients with advanced cirrhosis. Gastroenterology 2012;143:1253-60.

21. Zhang DL, Hao JY, Yang N. Value of D-dimer and protein $\mathrm{S}$ for diagnosis of portal vein thrombosis in patients with liver cirrhosis. J Int Med Res 2013;41:664-72.

22. Zhang D, Hao J, Yang N. Protein C and D-dimer are related to portal vein thrombosis in patients with liver cirrhosis. J Gastroenterol Hepatol 2010;25:116-21.

23. Englesbe MJ, Kubus J, Muhammad W, et al. Portal vein thrombosis and survival in patients with cirrhosis. Liver Transpl. 2010;16:83-90. 


\section{Supplementary}

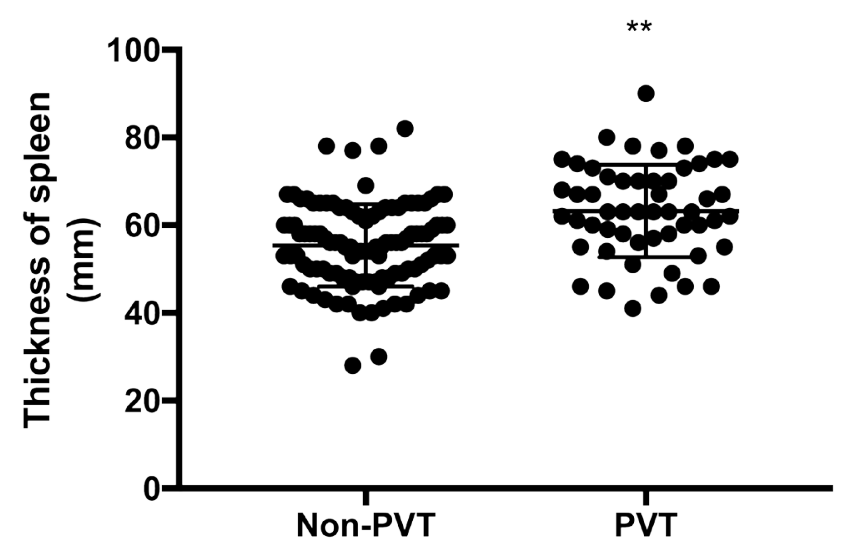

Figure S1 PVT increased the thickness of spleen. **, $\mathrm{P}<0.05$. 